\title{
TerraHS: Integration of Functional Programming and Spatial Databases for GIS Application Development
}

\author{
Sérgio Souza Costa ${ }^{1}$, Gilberto Câmara ${ }^{1}$, Danilo Palomo \\ ${ }^{1}$ Divisão de Processamento de Imagens (DPI) - Instituto Nacional de Pesquisas \\ Espaciais (INPE) \\ Av dos Astronautas, 1758 - 12227-001 - São José dos Campos - SP - Brasil \\ \{scosta,gilberto, danilo\}@dpi.inpe.br

\begin{abstract}
Recently, researchers in GIScience argued about the benefits on using functional programming for geospatial application development and prototyping of novel ideas. This paper presents an application that interfaces a functional language with a spatial database. It enables developing GIS applications development in a functional language, while handling data are in a spatial database. We used this application develop a Map Algebra, that shows the benefits on using this paradigm in GIScience. Our work shows there are many gains in using a functional language, especially Haskell, to write concise and expressive GIS applications. The TerraHS application allows a good compromise between the expressive power of a functional language, and the data handling facilities of an imperative language.
\end{abstract}

\section{Introduction}

Recent, research in GIScience proposes to use functional programming for geospatial application development [Frank and Kuhn 1995; Frank 1997; Frank 1999; Medak 1999; Winter and Nittel 2003]. Their main argument is that many of theoretical problems in GIScience can be expressed as algebraic theories. For these problems, functional languages enable fast development of rigorous and testable solutions [Frank and Kuhn 1995]. However, developing a GIS in a functional language is not feasible, since many parts needed for a GIS are already avaliable in imperative languages such as $\mathrm{C}++$ and Java. This is especially true for spatial databases, where applications such as PostGIS/PostgreSQL offer a basic support for spatial data management. It is unrealistic to develop such support using functional programming.

It is easier to benefit from functional programming for GIS application development if we build an application on top of an existing spatial database programming environment. This work presents TerraHS, an application that enables developing geographical applications in a functional language, using the data handling provided by TerraLib. TerraLib is a $\mathrm{C}++$ library that supports different spatial database management systems, and that includes many spatial algorithms. As a result, we get a combination of the good features of both programming paradigms.

This paper describes the TerraHS application. We briefly review the literature on functional programming and its use for GIS application development in Section 2. We describe how we built TerraHS in Section 3. In Section 4, we show the use of TerraHS for developing a Map Algebra. 


\section{Brief Review of the Literature}

\subsection{Functional Programming}

Functional programming is a programming paradigm that considers that computing is evaluating off mathematical functions. Functional programming stresses functions, in contrast to imperative programming, which stresses changes in state and sequential commands [Hudak 1989]. Recent functional languages include Scheme, ML, Miranda and Haskell. TerraHS uses the Haskell programming language. The Haskell report describes the language as:

"Haskell is a purely functional programming language incorporating many recent innovations in programming language design. Haskell provides higher-order functions, non-strict semantics, static polymorphic typing, user-defined algebraic datatypes, pattern-matching, list comprehensions, a module system, a monadic I/O system, and a rich set of primitive datatypes, including lists, arrays, arbitrary and fixed precision integers, and floating-point numbers" [Jones 2002].

The next section provides a brief description of the Haskell syntax. This description will help the reader to understand the essential arguments of this paper. For detailed description of Haskell see [Jones 2002], [Peyton Jones, Hughes et al. 1999] and [Thompson 1999].

\subsection{A Brief Tour of the Haskell Syntax}

Functions are the core of Haskell. A simple example is a function that which adds its two arguments:

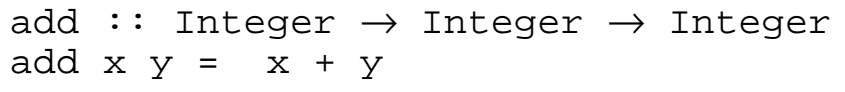

The first line defines the add function. It takes two Integer values as input and produces a third one. Functions in Haskell can also have generic (or polymorphic) types. For example, the following function calculates the length of a generic list, where [a] is a list of elements of a generic type a, [ ] is the empty list, and ( $\mathrm{x}: \mathrm{xs}$ ) is the list composition operation:

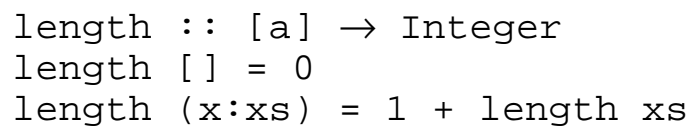

This definition reads "length is a function that calculates an integer value from a list of a generic type a. Its definition is recursive. The length of an empty list is zero. The length of a nonempty list is one plus the length of the list without its first element".

The user can define new types in Haskell using a data declaration, which defines a new type, or the type declaration, which redefines an existing type. For example, take the following definitions:

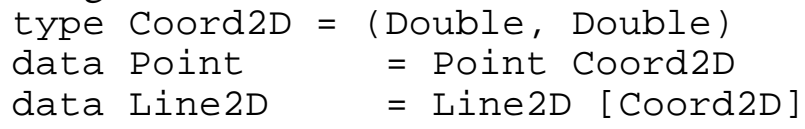

In these definitions, a Coord2D type is a shorthand for a pair of Double values. A point is a new type that contains one Coord2D. A Line2D is a new type that 
contains a list of Coord2D. One important feature of Haskell lists is that they can be defined by a mathematical expression similar to a set notation. For example, take the expression:

$$
\text { [elem } \mid \text { elem <- (domain map), (predicate elem obj)] }
$$

It reads "the list contains the elements of a map that satisfy a predicate that compares each element to a reference object". This expression could be used to select all objects that satisfy a topological operator ("all roads that cross a city"). Haskell includes higher-order functions. These are functions that have other functions as arguments. For example, the map higher-order function applies a function to a list, as follows:

$$
\begin{array}{ll}
\operatorname{map}::(a \rightarrow b) & \rightarrow[a] \rightarrow[b] \\
\operatorname{map} f[] & =[] \\
\operatorname{map} f(x: x s) & =f x: \operatorname{map} f x s
\end{array}
$$

This definition can reads as "take a function of type $a \rightarrow b$ and apply it recursively to a list of $a$, getting a list of $b$ ". Haskell supports overloading using type classes. A definition of a type class uses the keyword class. For example, the type class Eq provides a generic definition of all types that have an equality operator:

$$
\begin{aligned}
& \text { class Eq a where } \\
& (==):: \text { a } \rightarrow \text { a } \rightarrow \text { Bool }
\end{aligned}
$$

This declaration reads "a type a is an instance of the class Eq if it defines is an overloaded equality (==) function." We can then specify instances of type class Eq using the keyword instance. For example:

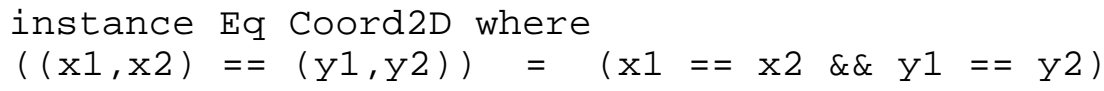

Haskell also supports a notion of class extension. For example, we may wish to define a class ord which inherits all the operations in Eq, but in addition includes comparison, minimum and maximum functions:

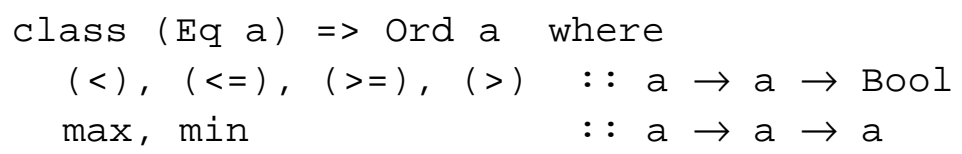

\subsection{Functional Programming and GIS}

Many recent papers propose using functional languages for GIS application develepment [Frank and Kuhn 1995; Frank 1997; Frank 1999; Winter and Nittel 2003]. Frank and Kuhn [1995] show the use of functional programming languages as tools for specification and prototyping of Open GIS specifications. Winter and Nittel [2003] apply a formal tool to writing specifications for the Open GIS proposal for coverages. Medak [1999] develops an ontology for life and evolution of spatial objects in an urban cadastre. To these authors, functional programming languages satisfy the key requirements for specification languages, having expressive semantics and allowing rapid prototyping. Translating formal semantics is direct, and the resulting algebraic structure is extendible. However, these works do not deal with issues related to $\mathrm{I} / \mathrm{O}$ and to database management. Thus, they do not provide solutions applicable to real-life problems. To 
apply these ideas in practice, we need to integrate functional and imperative programming.

\subsection{Integration of Functional and Imperative Languages}

The integration functional and imperative languages is discussed in Chakravarty [2003], who presents the Haskell 98 Foreign Function Interface (FFI), which supports calling functions written in $\mathrm{C}$ from Haskell and vice versa. However, functions written in imperative languages can contain side effects. To allow functional languages to deal with side effects, Wadler [1990] proposed monads for structuring programs written in functional language. The use of monads enables a functional language to simulate an imperative behavior with state control and side effects [Thompson 1999]. Jones [2005] presents many crucial issues about interaction of functional languages with the external world, such as I/O, concurrency, exceptions and interfaces to libraries written in other languages. In this work, the author describes a Haskell web server as a case study. These works show of the integration between these two programming styles. However, none of these works deals with geoinformation systems. On the next section we present an application that integrates programs written in Haskell with spatial databases and allows fast and reliable GIS application development.

\section{TerraHS}

This section presents TerraHS, a software application which enables developing geographical applications using in functional programming using data stored in a spatial database. TerraHS links the Haskell language to in the TerraLib GIS library. TerraLib is a class library written in $\mathrm{C}++$, whose functions provide spatial database management and spatial algorithms. TerraLib is free software [Vinhas and Ferreira 2005]. TerraHS links to the TerraLib functions through the Foreign Function Interface [Chakravarty 2003] and a function set written in $\mathrm{C}$ language, which performs the TerraLib functions. The Figure 1 shows its architecture.

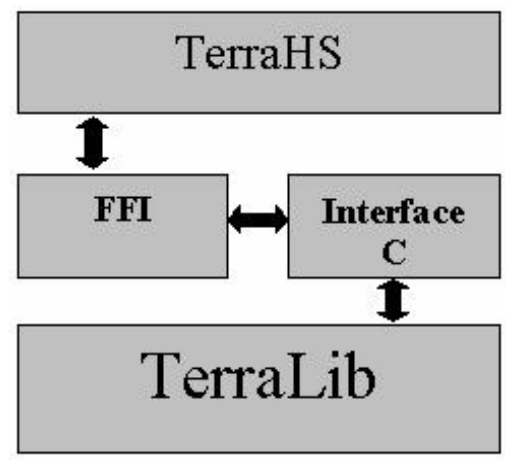

Figure 1 TerraHS Architecture

TerraHS includes three basic resources for geographical applications: spatial representations, spatial operations and database access. The next sections present them. 


\subsection{Spatial Representations}

\subsubsection{Vector data structures}

Identifiable entities on the geographical space, or geo-objects, such as cities, highways or states are usually represented in vector data structures, as point, line and polygon. These data structures represent an object by one or more pairs of Cartesian coordinates. TerraLib represents coordinate pairs through the Coord2D data type. In TerraHS, this type is a tuple of real values.

type Coord2D $=$ (Double, Double)

The type Coord2D is the basis for all the geometric types in TerraHS, namely:

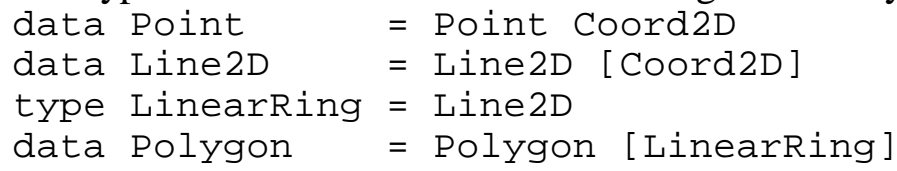

The Point data type represents a point in TerraHS, and is a single instance of a Coord2D. The Line $2 D$ data type represents a line, composed of one or more segments and it is a vector of Coord2Ds [Vinhas and Ferreira 2005]. The LinearRing data type represents a closed polygonal line. This type is a single instance of a Line $2 D$, where the last coordinate is equal to the first [Vinhas and Ferreira 2005]. The Polygon data type represents a polygon in TerraLib, and it is a list of LinearRing. Other data types include:

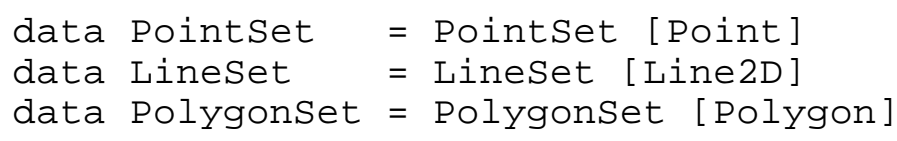

\subsubsection{Cell-Spaces}

TerraLib supports cell spaces. Cell spaces are a generalized raster structure where each cell stores a more than one attribute value or as a set of polygons that do not intercept one another. A cell space enables joint storage of the entire set of information needed to describe a complex spatial phenomenon. This brings benefits to visualization, algorithms and user interface [Vinhas and Ferreira 2005]. A cell contains a bounding box and a position given by a pair of integer numbers.

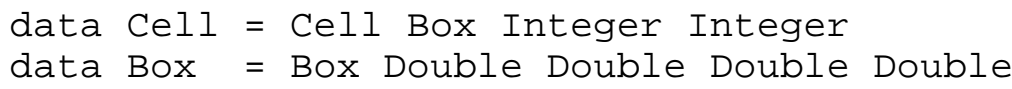

The Box data type represents a bounding box and the Cell data type represents one cell in the cellular space. The CellSet data type represents a cell space.

data Cellset $=$ Cellset $[\mathrm{Cell}]$

\subsubsection{Spatial Operations}

TerraLib provides a set of spatial operations over geographic data. TerraHS provides function that use those algorithms. We used Haskell type classes [Shields and Jones 2001; Chakravarty 2004] to define the spatial operations using polymorphism. These topologic operations can be applied for any combination of types, such as point, line and polygon.

\section{class Topologyops a b where}




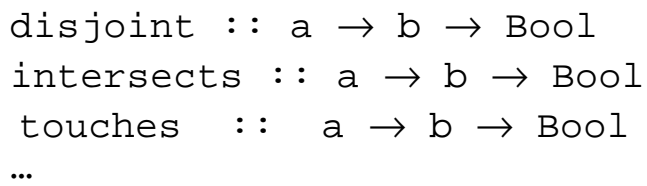

The TopologyOps class defines a set of generic operations, which can be instantiated to several combinations of types:

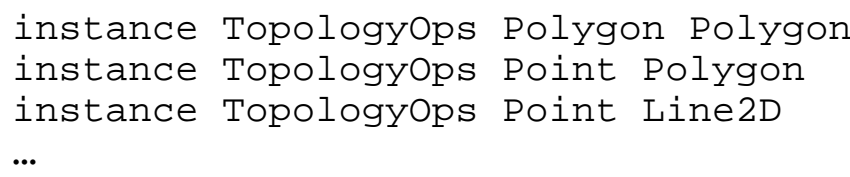

\subsection{Database Access}

One of the main features of TerraLib is its use of different object-relational database management systems (OR-DBMS) to store and retrieve the geometric and descriptive parts of spatial data [Vinhas and Ferreira 2005]. TerraLib follows a layered model of architecture, where it plays the role of the middleware between the database and the final application. Integrating Haskell with TerraLib enables an application developed in Haskell to share the same data with applications written in $\mathrm{C}++$ that use TerraLib, as shown in Figure 2.

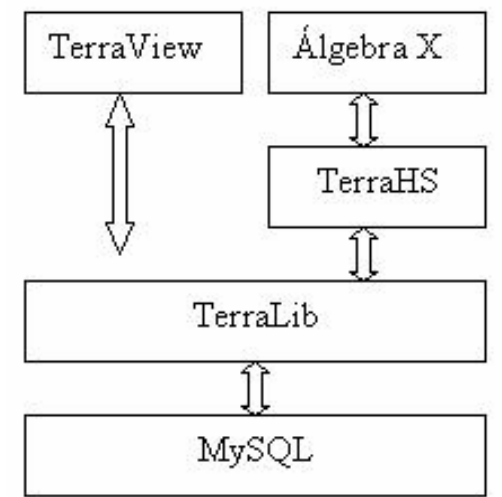

Figure 2 - Using the TerraLib to share a geographical database, adapted from Vinhas e Ferreira (2005).

A TerraLib database access does not depends on a specific DBMS and uses an abstract class called TeDatabase [Vinhas and Ferreira 2005]. In TerraHS, the database classes are algebraic data types, where each constructor represents a subclass.

data Database $=$ MySQL String String String string

| PostgreSQL String String String String

A TerraLib layer aggregates spatial information located over a geographical region and that share the same attributes. A layer is identifier in a TerraLib database by its name [Vinhas and Ferreira 2005].

type LayerName $=$ String

In TerraLib, a geo-object is an individual entity that has geometric and descriptive parts, composed by:

- Identifier: identifies a geo-object. 
data ObjectId = ObjectId String

- Attributes: this is the descriptive part of a geo-object. An attribute has a name (AttrName) and a value (Value).

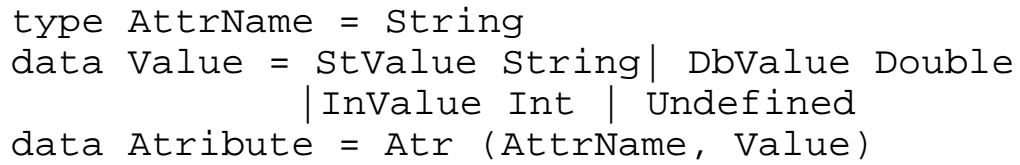

- Geometries: this is the spatial part, which can have different representations.

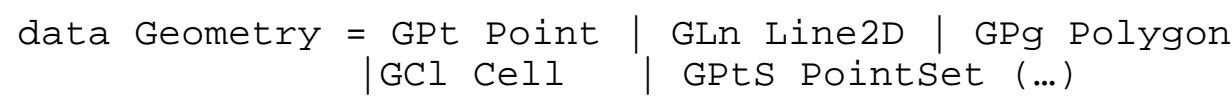

A geo-object in TerraHS is a triple:

data Geobject $=$ Geoobject (ObjectId, [Atribute], Geometry)

The GeoDatabases type class provides generic functions for storage, retrieval of geo-objects from a spatial database.

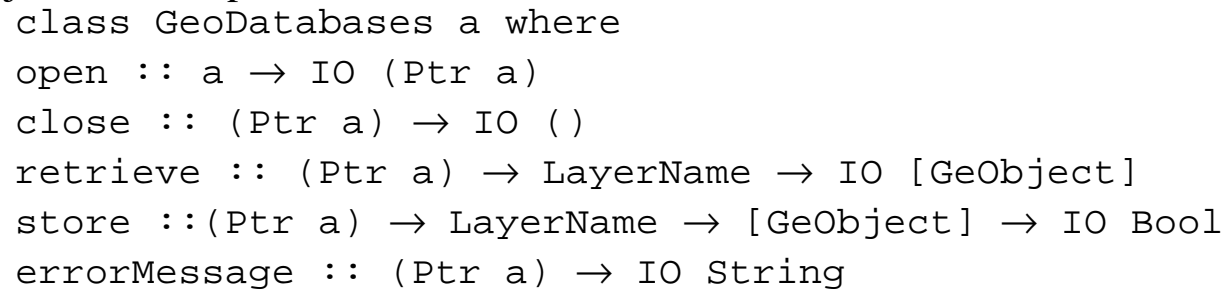

These operations will then be instantiated to a specific database, such as mySQL or PostgreSQL. Figure 3 shows an example of a TerraLib database access program.

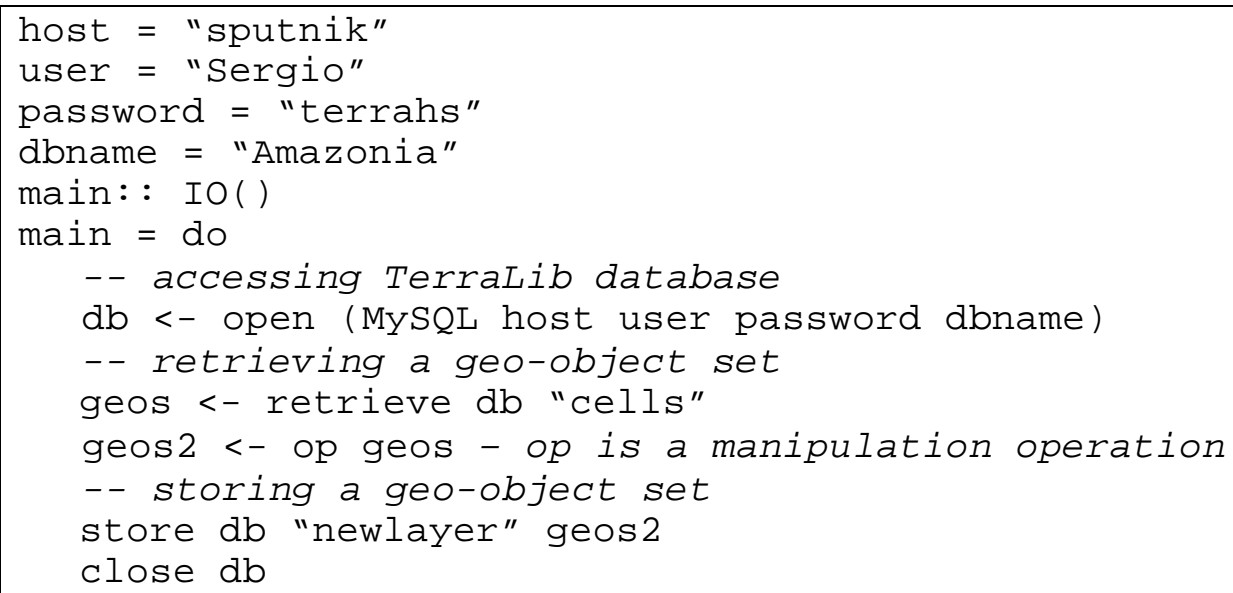

Figure 3 - Acessing a TerraLib database using TerraHS

\section{A generalized map algebra}

One of the important uses of functional language for GIS is to enable fast and sound development of new applications. As an example, this section presents a map algebra in a functional language. In GIS, maps are a continuous variable or to a categorical classification of space (for example, soil maps). Map Algebra is a set of procedures for 
handling maps. They allow the user to model different problems and to get new information from the existing data set. The main contribution to map algebra comes from the work of Tomlin [1983]. Tomlin's model uses a single data type (a map), and defines three types of functions. Local functions involve matching locations in different map layers, as in "classify as high risk all areas without vegetation with slope greater than 15\%". Focal functions involve proximal locations in the same layer, as in the expression "calculate the local mean of the map values". Zonal functions summarize values at locations in a layer contained in zones defined in another layer. An example is "given a map of city and a digital terrain model, calculate the mean altitude for each city."

For this experiment, we use the map algebra proposed in Câmara et al. [Câmara 2005]. The authors describe the design of a map algebra that generalizes Tomlin's map algebra by incorporating topological and directional spatial predicates. In the next section, we describe and implement this algebra.

\subsection{The map abstract data type}

Our map algebra has two main data types: object set and field. An object set is a set of objects represented by points, lines or regions associated with nonspatial attribute. Fields are functions that map a location in a spatial partition to a nonspatial attribute. The map data type combines both the object set data type and the field data type. A map is a function $m:: E \rightarrow A$, where:

- The domain is finite collection, either a set of cells or a set of objects.

- The range is a set of attribute values.

For each geographic element $e \in E$, a map returns a value $m(e)=a$, where $a \in$ A. A geographical element can represent a location, area, line or point. This definition matches the definition of a coverage in Open GIS [OGC 2000]. A coverage in a planarenforced spatial representation that covers a geographical area completely and divides it in spatial partitions that may be either regular or irregular. For retrieving data from a coverage, the Open GIS specification propose describes a discrete function (DiscreteC_Function), as shown in Figure 4 below. 


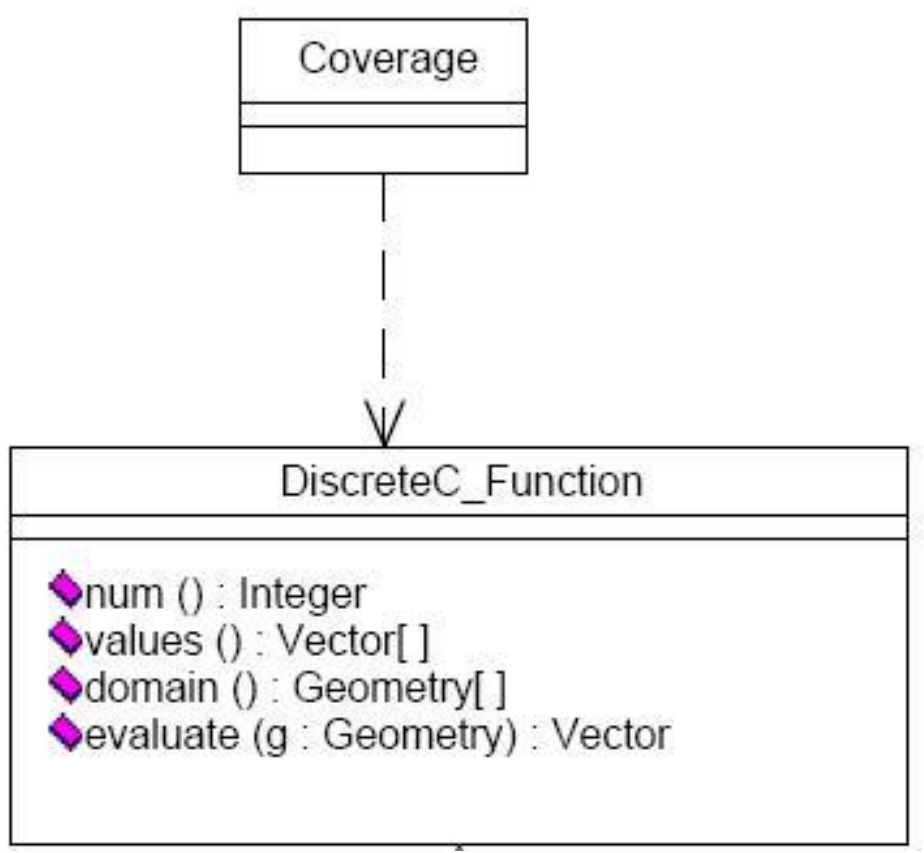

Figure 4 The Open GIS discrete coverage function - source: [OGC 2000].

The DiscreteCFunction data type describes a function whose spatial domain and whose range are finite. The domain consists of a finite collection of geometries, where a DiscreteCFunction maps each geometry for a value [OGC 2000]. Based on the Open GIS specification, we defined the type class Maps. The type class Maps generalizes and extends the DiscreteCFunction class. Its functions are parameterized on the input type a and the output type b. It provides the support for the operations proposed by the DiscreteCFunction:

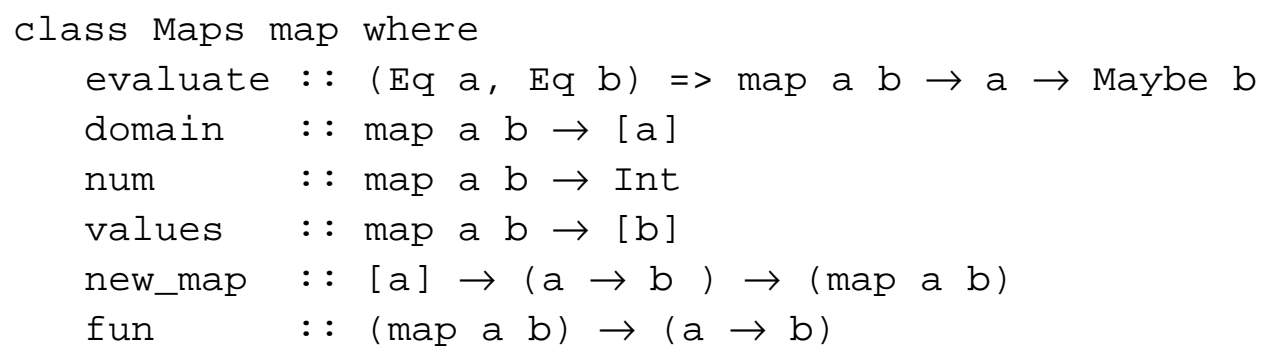

The functions is the Maps type class work as follows: (a) evaluate is a function that takes a map and an input value a and produces an output value ("give me the value of the map at location a"); (b) domain is a function that takes a map and returns the values of its domain; (c) num returns the number of elements of the map's domain; (d) values returns the values of the map's range. We propose two extra functions: new_map and fun, as described below.

- new_map, a function that returns a new map $m$, given a domain and a coverage function.

- fun: given a map, returns its coverage function.

We defined the Map data type to use the functions of the generic type class Maps. The Map data type is also parameterized. 
data Map a b $\operatorname{Map}((\mathrm{a} \rightarrow \mathrm{b}),[\mathrm{a}])$

The data type Map has two parts:

- A coverage function that maps an object of generic type $a$ to generic type $b$.

- A domain of objects of the polymorphic type $a$.

The instance of the type class Maps to the Map data type is shown below:

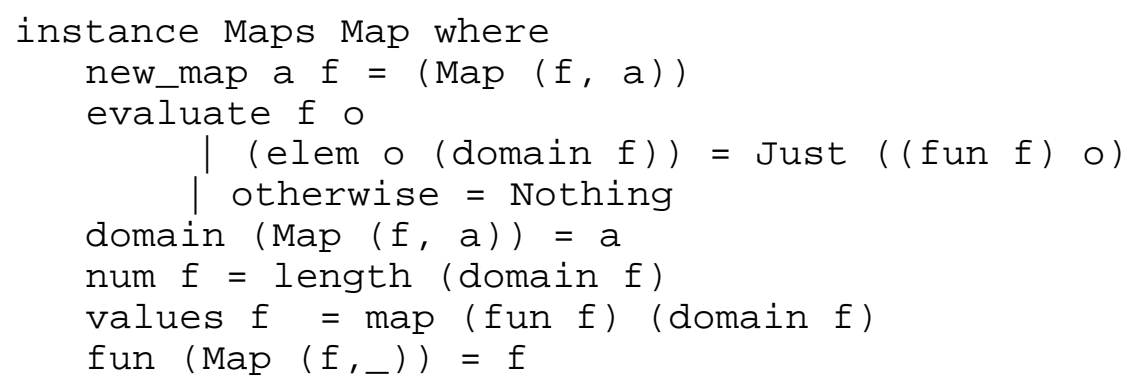

Figure 5 show an example of the Map data type.

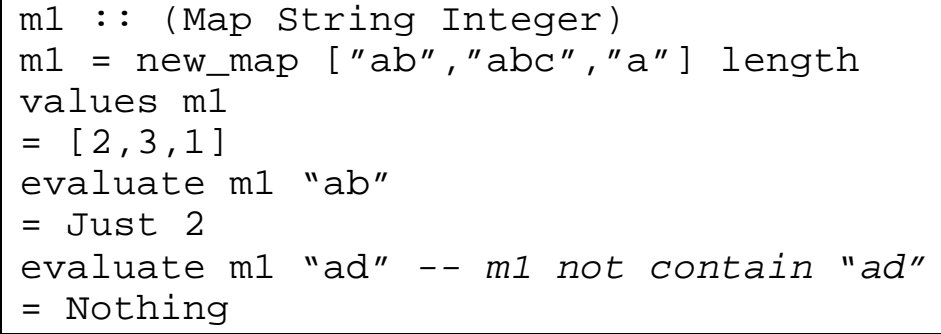

Figure 5 Example of use of the Map data type.

\subsection{Operations}

Câmara et al [2005] define two classes of the map algebra operations: nonspatial and spatial. For nonspatial operations, the value of a location in the output map is obtained from the values of the same location in one or more input maps. They include logical expressions such as "classify as high risk all areas without vegetation with slope greater than 15\%", "Select areas higher than 500 meters", "Find the average of deforestation in the last two years", and "Select areas higher than 500 meters with temperatures lower than 10 degrees". Spatial functions are those where the value of a location in the output map is computed from the values of the neighborhood of the same location in the input map. They include expressions such as "calculate the local mean of the map values" and "given a map of cities and a digital terrain model, calculate the mean altitude for each city". In what follows, we show these operations in TerraHS, using polymorphic data types. 


\subsubsection{Nonspatial operations}

Nonspatial operations are higher-order functions that take one value for each input map and produce one value in the output map, using a first-order function as argument. These include single argument functions and multiple argument functions [Câmara, Palomo et al. 2005].

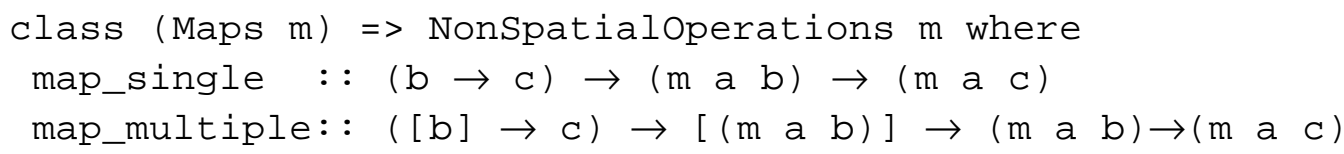

The map_single function has two arguments: a map $m$ and a first-order function $g$. It returns a new map, whose domain contains the same elements of the input map domain. The coverage function of the output map is the composition of the coverage function of the input map $m$ and the first-order function $g$.

map_single $g \mathrm{~m}=$ new_map (domain $\mathrm{m}$ ) ( $\mathrm{g} \cdot($ fun $\mathrm{m}$ ))
\begin{tabular}{|l|l|l|l|}
$\begin{array}{l}\text { defines a new map } \\
\text { with the same domain }\end{array}$ & $\begin{array}{l}\text { defines the mapping } \\
\text { function of the new map }\end{array}$ \\
\hline
\end{tabular}

Figure 6 shows an example of a single argument function.

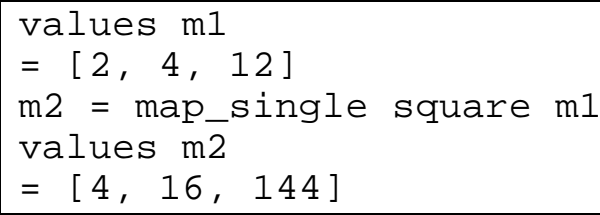

Figure 6 Example of use of the single argument function

The map_multiple function has three arguments: a map list, a multivalued function and a reference map. Given a reference map, it applies a multivalued function in map list.

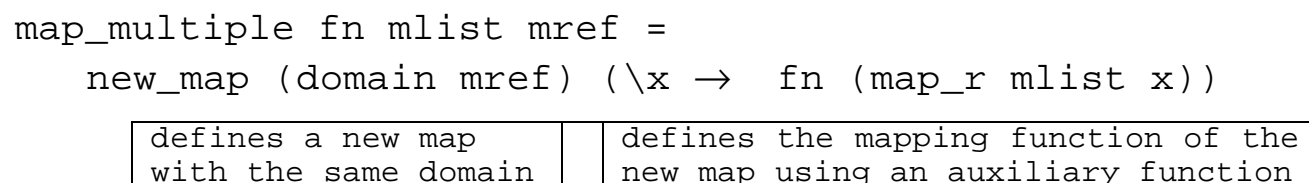

The map_multiple function returns a new map with a same domain of the reference map and a new coverage function. This function uses the auxiliary function map_r. For each element $x$ of the reference map, map_r applies the multiargument function in the input list of maps to get the output value. It also handles cases where there are multiargument function fails to returns an output value.

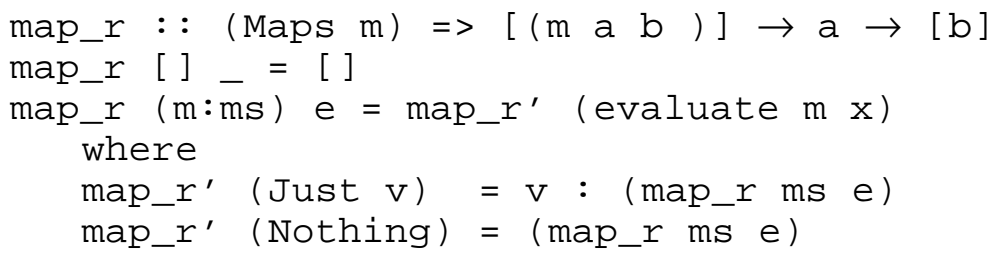

Figure 7 shows an example of map_multiple. In this example, the m3 map is the result of the sum of the maps $m 1$ and $m 2$. 


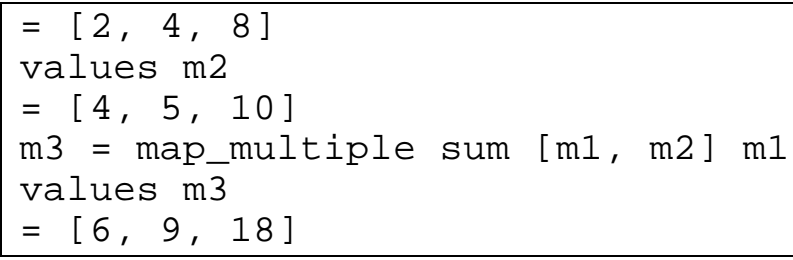

Figure 7 - Example of use of map_multiple

\subsubsection{Spatial Operations}

Spatial operations are higher-order functions that use a spatial predicate. These functions combine a selection function and a multivalued function, with two input maps (the reference map and the value map) and an output map [Câmara, Palomo et al. 2005]. Spatial functions generalize Tomlin's focal and zonal operations and have two parts: selection and composition. For each location in the output map, the selection function finds the matching region on the reference map. Then it applies the spatial predicate between the reference map and the value map and creates a set of values. The composition function uses the selected values to produce the result (Figure 8). Take the expression "given a map of cities and a digital terrain model, calculate the mean altitude for each city”. In this expression, the value map is the digital terrain model and the reference map is the map of cities. The evaluation has two parts. First, it selects the terrain values inside each city. Then, it calculates the average of these values.

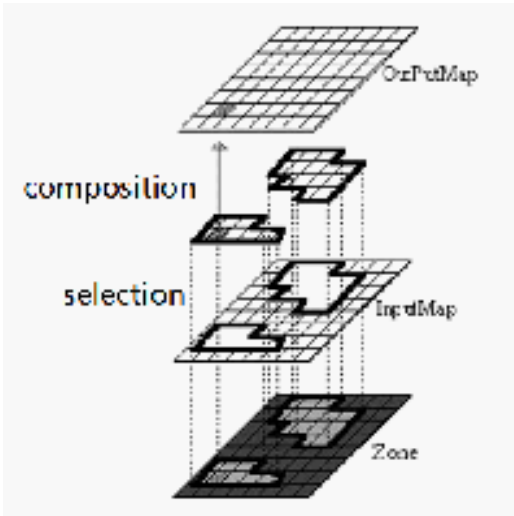

Figure 8. Spatial operations (selection + composition). Adapted from Tomlin [1990].

The implicit assumption is that the geographical area of the output map is the same as reference map. The type signature of the spatial functions in TerraHS is:.

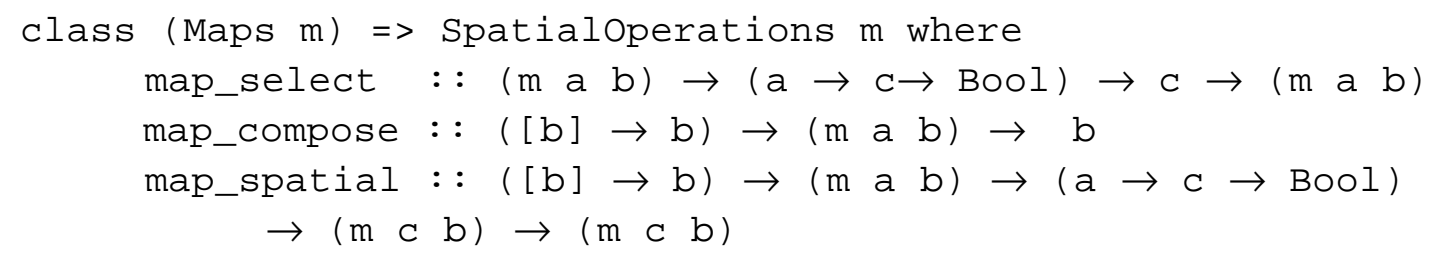

The spatial selection function selects all elements that satisfy a predicate on a reference object ("select all deforested areas inside the state of Amazonas"). It has three arguments: an input map, a predicate and a reference element.

map_select $m$ pred obj = new_map sel_dom (fun m) 
where

sel_dom $=$ [elem $\mid$ elem $\leftarrow$ (domain $m),($ pred elem obj) $]$

This function takes a reference element and an input map. It creates a map that contains all elements of the input map that satisfy the predicate over the reference element. Figure 9 shows an example, where the map consists of a set of points. Then, we select those points that intersect a given line.

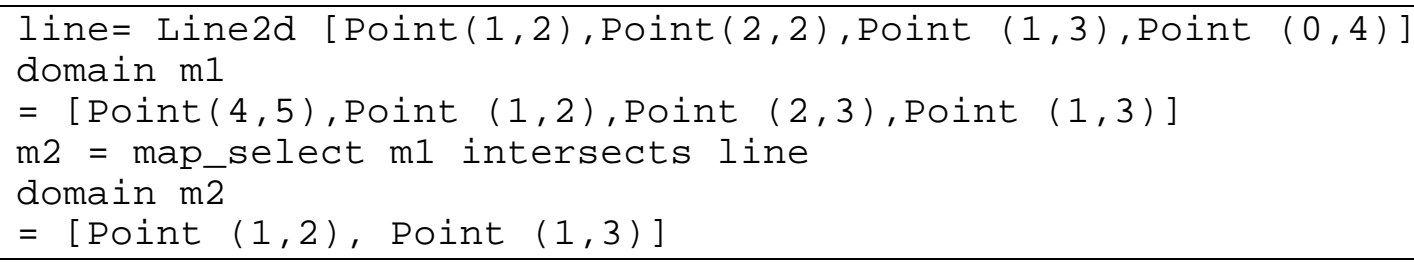

Figure 9 Example of map_select.

The composition function combines selected values using a multivalued function. In Figure 10, the map_compose function is applied to map $m l$ and to the multivalued function sum.

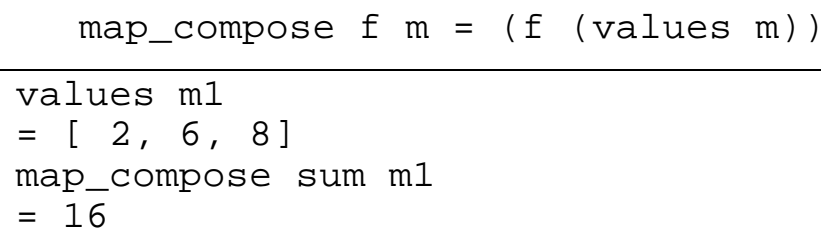

Figure 10 Example of map_compose.

The map_spatial function combines spatial selection and spatial composition:

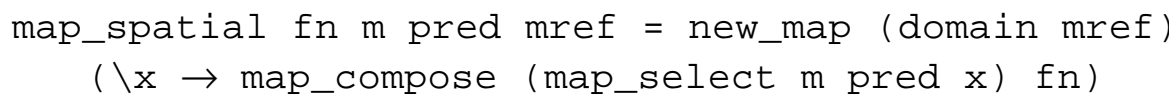

Map_spatial creates a map whose domain contains the elements of the reference map. To get its coverage function, we apply map_compose to the result of the map_selection. Figure 12 shows an example.

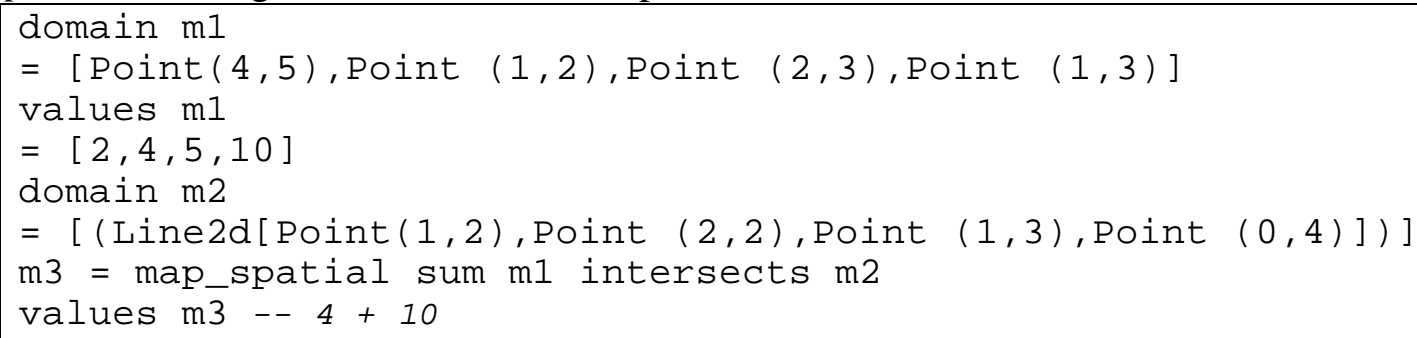

Figure 12 Example of map_spatial

The spatial operation selects all points of $m 1$ that intersect $m 2$ (which is a single line). Then, it sums its values. In this case, points $(1,2)$ and $(1,3)$ intersect the line. The sum of their values is 14 . 


\subsection{Application Examples}

In the previous section we described how to express the map algebra proposed in Câmara et al. [2005] in TerraHS. In this section we show the application of this algebra to actual geographical data.

\subsubsection{Storage and Retrieval}

Since a Map is generic data type, it can be applied to different concrete types. In this section we apply it to the Geometry and Value data types available in the TerraHS, which represent, respectively, a region and a descriptive value. TerraHS enables storage and retrieval of a geo-object set. To perform a map algebra, we need to convert from a geoobject set to a map and vice versa.

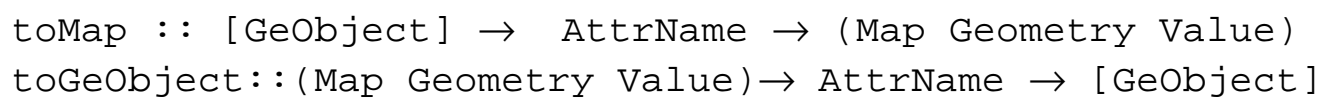

Given a geo-object set and the name of one its attributes, the toMap function returns a map. Remember that a Map type has one value for each region. Thus, a layer with three attributes it produce three Maps. The toGeObject function inverts the tomap function. Details of these two functions are outside the scope of this paper. Given these functions, we can store and retrieve a map, given a spatial database.

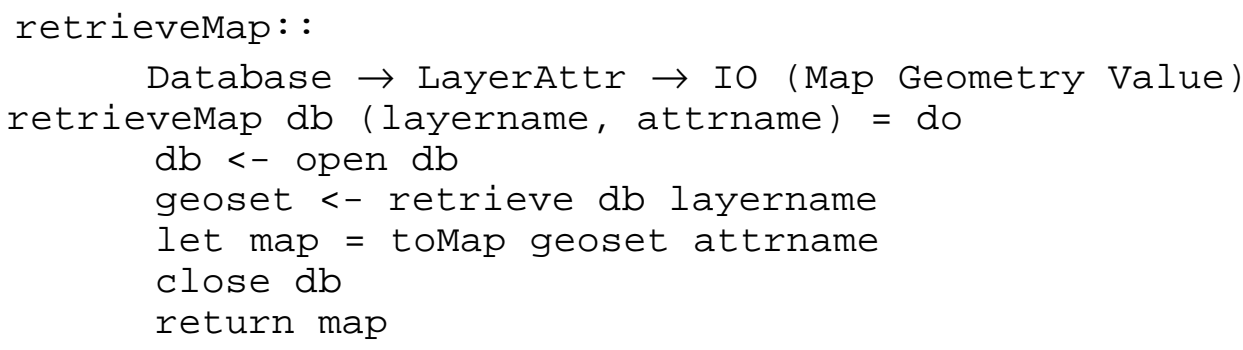

The LayerAttr type is a tuple that represents the layer name and attribute name. The retrievemap function connects to the database, loads a geo-object set, converts these geo-objects into a map, and return this map as its output.

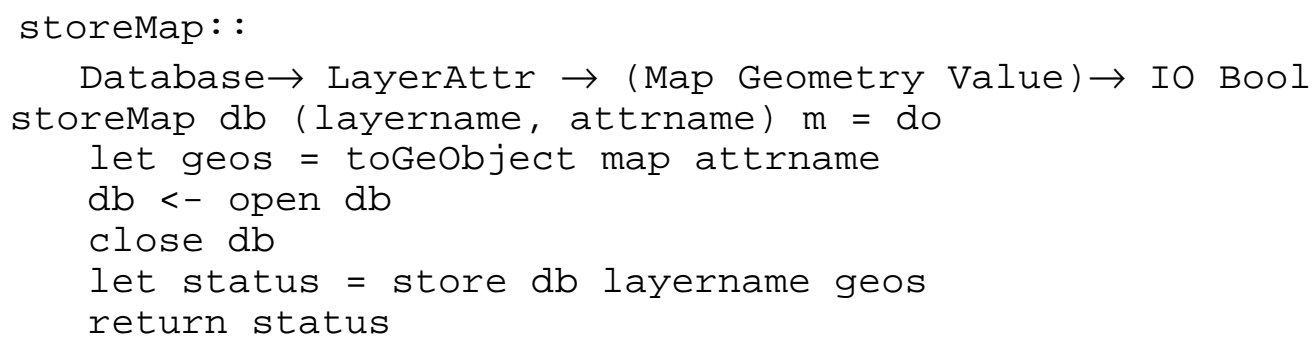

The storemap function coverts a map to a geo-object set that will be saved in the database. We can now write a program that reads and writes a map in a TerraLib database. 


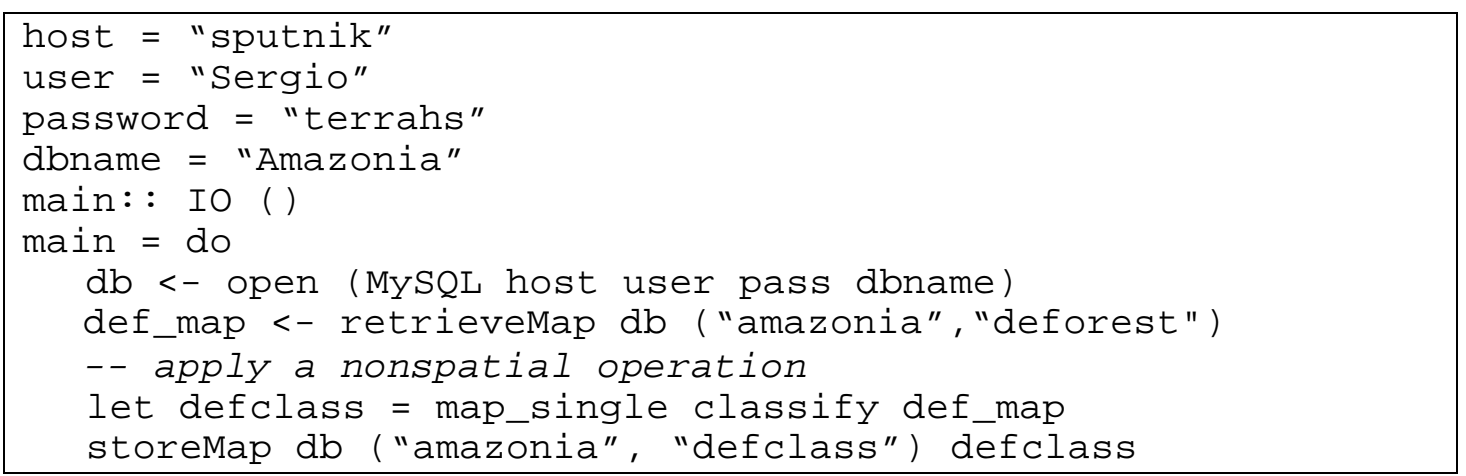

Figure 13 Retrieving and storing a Map from TerraLib Database

\subsubsection{Examples of Map Algebra in TerraHS}

Since 1989, the Brazilian National Institute for Space Research has been monitoring the deforestation of the Brazilian Amazon, using remote sensing images. We use some of this data as a basis for our examples. We selected a data set from the central area of Pará, composed by a group of highways and two protection areas. This area is divided in cells of $25 \times 25 \mathrm{~km} 2$, where each cell describes the percentage of deforestation and deforested area (Figure 14).

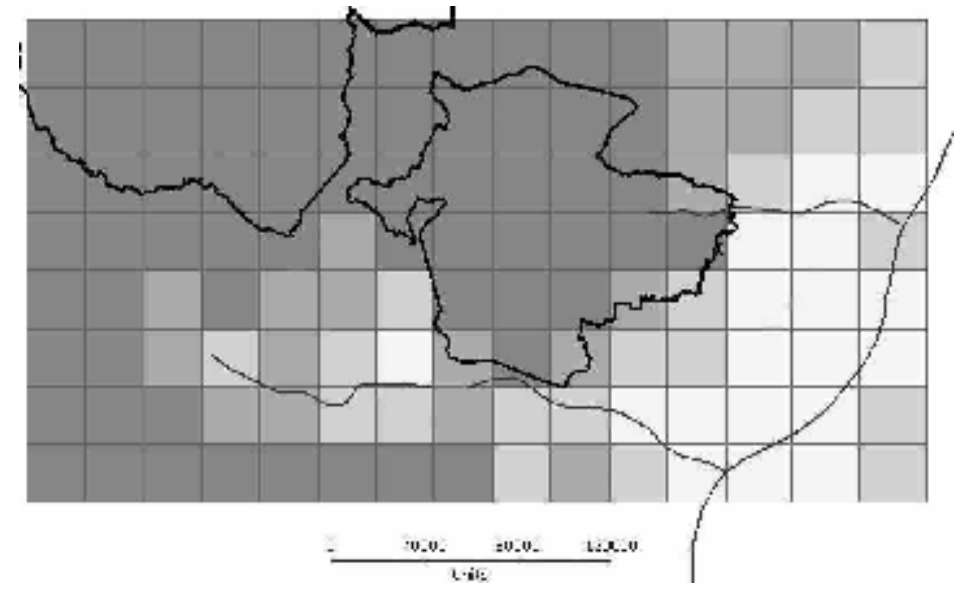

Figure 14 - Deforestation, Protection Areas and Roads Maps (Pará State)

Our first example considers the expression: "Given a map of deforestation and classification function, return the classified map". The classification function defines four classes: (1) dense forest; (2) mixed forest with agriculture; (3) agriculture with forest fragments; (4) agricultural area. This function is:

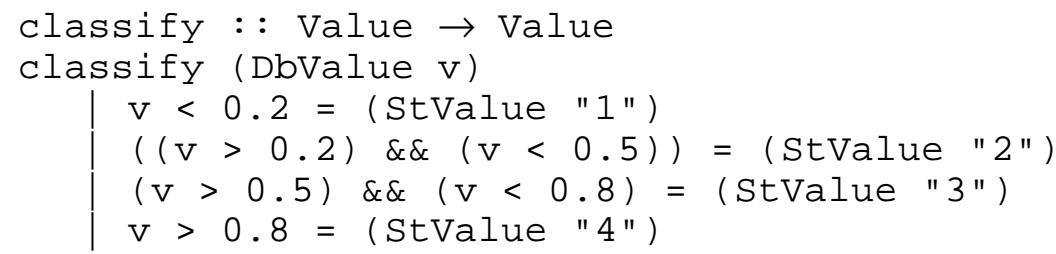

We obtain the classified map using the map_single operation together with the classify function: 


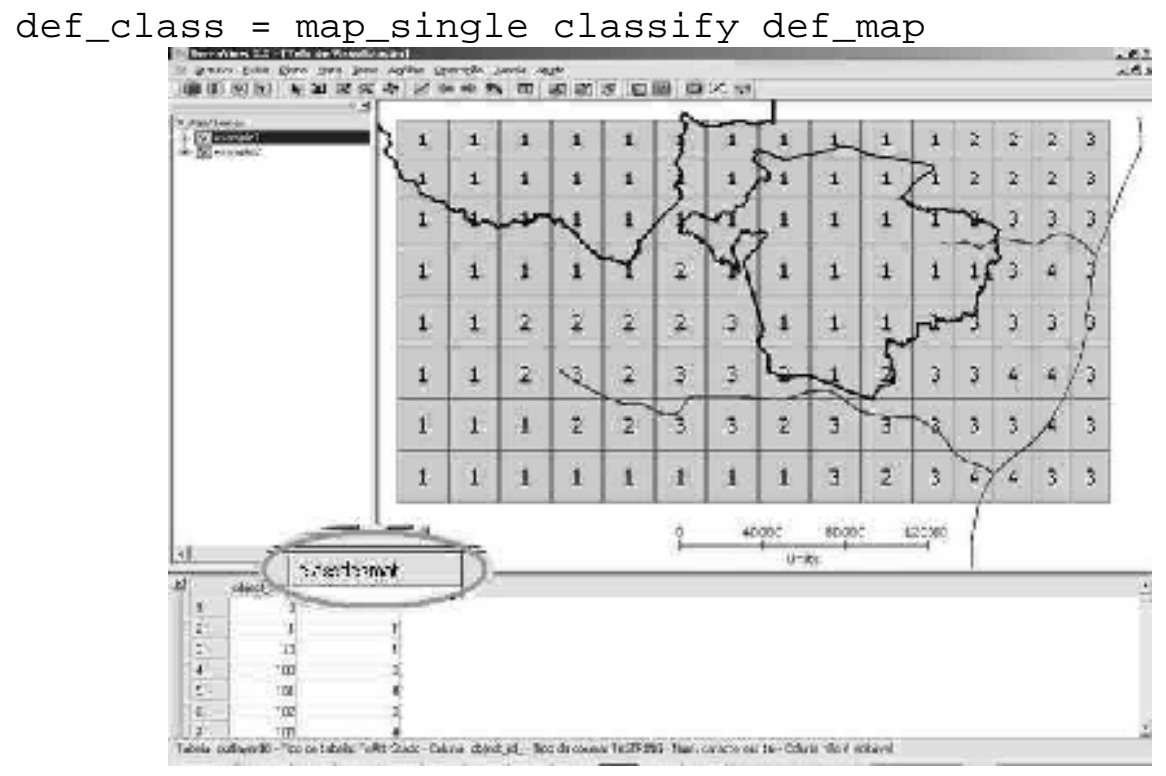

Figure 15 - The classified map

As a second example, we take the expression: "Calculate the mean deforestation for each protection area". The inputs are: the deforestation map (def_map), a spatial predicate (within), a multivalued function (mean) and the map of protected areas (prot_areas). The output is a deforestation map of the protected areas (def_prot) with the same objects as the reference map (prot_areas). We use the map_spatial higher-order operation to produce the output:

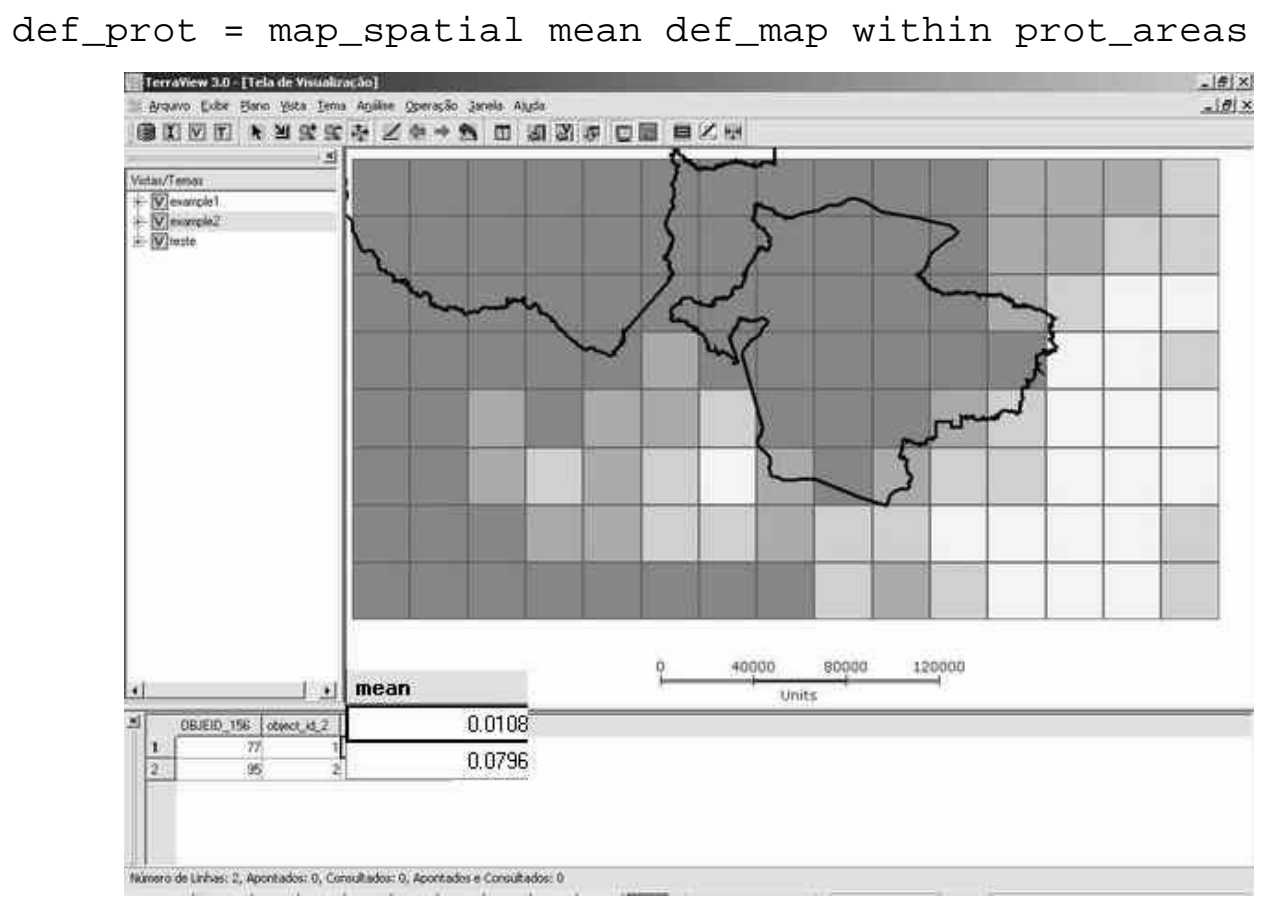

Figure 16 - Deforest mean by protection area

In our third example, we consider the expression: "Given a map containing roads and a deforestation map, calculate the mean of the deforestation along the roads". We have as inputs: the deforestation map (def_map), a spatial predicate (intersect), a 
multivalued function (mean) and a road map (roads). The product is a map with one value for each road. This value is the mean of the cells that intercept this road.

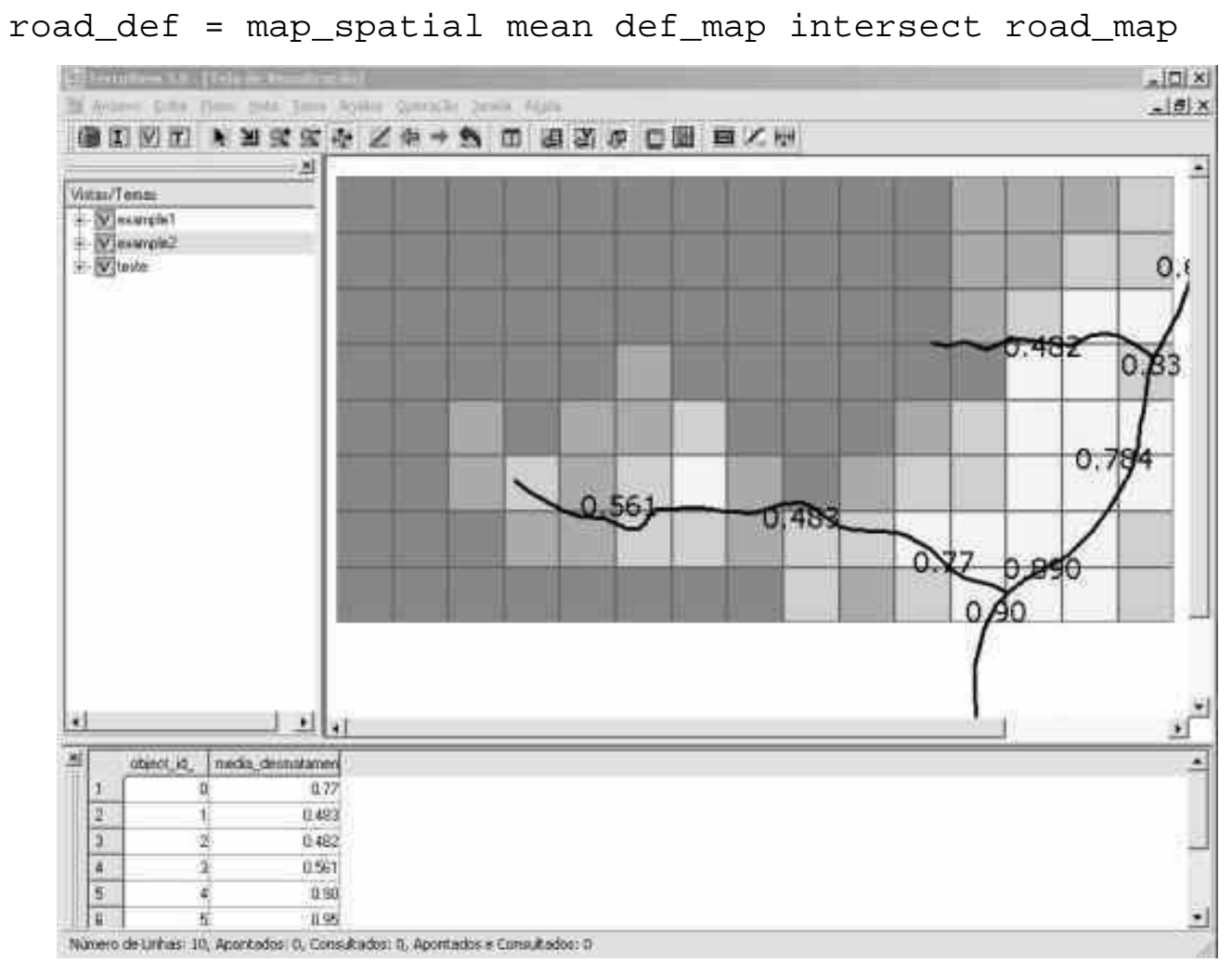

Figure 17 - Deforestation mean along the roads

\section{Conclusions}

This paper presents the TerraHS application for integrating functional programming and spatial databases. We use TerraHS to develop and validate a map algebra in a functional language. The resulting map algebra is compact, generic and extensible. The example shows the benefits on using functional programming, since it enables a fast prototyping and testing cycle. Table 1 presents the total number of Haskell lines used to develop the map algebra.

Table 1 - Map Algebra in Haskell

\begin{tabular}{|l|l|l|l|}
\hline \multirow{2}{*}{} & \multicolumn{3}{|c|}{ Number of source lines } \\
\cline { 2 - 4 } & operations & axioms & total \\
\hline Data types & 6 & 9 & 15 \\
\hline Map Algebra & 6 & 10 & 16 \\
\hline Auxiliary & 1 & 5 & 6 \\
\hline Total & 13 & 24 & 37 \\
\hline
\end{tabular}

For comparison purposes, the SPRING GIS [Câmara, Souza et al. 1996] includes a map algebra in the $\mathrm{C}++$ language that uses about 8,000 lines of code. The SPRING 
map algebra provides a strict implementation of Tomlin's algebra. Our map algebra allows a more generic set of functions than Tomlin's at less than $1 \%$ of the code lines. This large difference comes from the use of the parameterized types, overloading and higher order functions, which are features of the Haskell language. Our work points out that integrating functional languages with spatial database is an efficient alternative in for developing and prototyping novel ideas in GIScience.

\section{References}

Câmara, G. (2005). Representação computacional de dados geográficos. Bancos de Dados Geográficos. M. Casanova, G. Câmara, C. Davis, L. Vinhas and G. Ribeiro. Curitiba, MundoGeo Editora: 11-52.

Câmara, G., D. Palomo, R. C. M. d. Souza, et al. (2005). Towards a generalized map algebra: principles and data types. VII Workshop Brasileiro de Geoinformática, Campos do Jordão, SBC.

Câmara, G., R. Souza, U. Freitas, et al. (1996). "SPRING: Integrating Remote Sensing and GIS with Object-Oriented Data Modelling." Computers and Graphics 15(6): 13-22.

Casanova, M., G. Camara, C. Davis, et al., Eds. (2005). Bancos de Dados Geograficos (Spatial Databases). Curitiba, Editora MundoGEO.

Chakravarty, A. P. a. M. (2004). Interfacing Haskell with Object-Oriented Languages. 15th International Workshop on the Implementation of Functional Languages, Lübeck, Germany, Springer-Verlag.

Chakravarty, M. (2003). "The Haskell 98 foreign function interface 1.0: An addendum to the Haskell 98 report."

Frank, A. (1997). Higher order functions necessary for spatial theory development. AutoCarto 13, Seattle, WA, ACSM/ASPRS.

Frank, A. (1999). One Step up the Abstraction Ladder: Combining Algebras - From Functional Pieces to a Whole. COSIT - Conference on Spatial Information Theory, Springer-Verlag.

Frank, A. and W. Kuhn (1995). Specifying Open GIS with Functional Languages. Advances in Spatial Databases - 4th International Symposium, SSD '95, Portland, ME. M. Egenhofer and J. Herring. Berlin, Springer-Verlag. 951: 184195.

Hudak, P. (1989). "Conception, evolution, and application of functional programming languages." ACM Comput. Surv. 21(3): 359-411.

Jones, S. P. (2002). "Haskell 98 Language and Libraries The Revised Report."

Jones, S. P. (2005). "Tackling the Awkward Squad: monadic input/output, concurrency, exceptions, and foreign-language calls in Haskell."

Medak, D. (1999). Lifestyles - a new Paradigm in Spatio-Temporal Databases. Department for Geoinformation. Vienna, Technical University of Vienna. 
OGC. (2000). "Open GIS Consortium. Topic 6: the coverage type and its subtypes." Retrieved 10/05/2006, 2006, from http://portal.opengeospatial.org/files/?artifact id=7198.

Peyton Jones, S., J. Hughes and L. Augustsson. (1999). "Haskell 98: A Non-strict, Purely Functional Language." from http://www.haskell.org/onlinereport/.

Shields, M. and S. L. P. Jones (2001). "Object-Oriented Style Overloading for Haskell." Electronic Notes in Theoretical Computer Science 59(1).

Thompson, S. (1999). Haskell:The Craft of Functional Programming. Harlow, England, Pearson Education.

Tomlin, C. D. (1983). A Map Algebra. Harvard Computer Graphics Conference. Cambridge, MA.

Vinhas, L. and K. R. Ferreira (2005). Descrição da TerraLib. Bancos de Dados Geográficos. M. Casanova, G. Câmara, C. Davis, L. Vinhas and G. Ribeiro. Curitiba, MundoGeo Editora: 397-439.

Wadler, P. (1990). Comprehending monads. Proceedings of the 1990 ACM conference on LISP and functional programming \%@ 0-89791-368-X. Nice, France, ACM Press: 61-78.

Winter, S. and S. Nittel (2003). "Formal information modelling for standardisation in the spatial domain." International Journal of Geographical Information Science 17: 721--741. 